\title{
Effets de la toxicité des pesticides Maneb et Chlorpyrifos-Ethyl sur un poisson d'eau douce, Oreochromis niloticus
}

\author{
Lévis GAPESSIE NTIENDJUI ${ }^{1 *}$, Simon Awafor TAMUNGANG ${ }^{1}$, \\ Ferdinand NGOULA ${ }^{2}$, Gilbert ATEUFACK ${ }^{3}$ et Joseph TCHOUMBOUE ${ }^{2}$ \\ ${ }^{1}$ Université de Dschang, Faculté des sciences, Département de Biologie Animale, Laboratoire \\ d'écologie appliquée, B.P.67 Dschang-Cameroun. \\ ${ }^{2}$ Université de Dschang, Faculté d'Agronomie et des sciences Agricoles, Département des productions \\ Animales, Laboratoire de physiologie Animale, B.P. 222 Dschang-Cameroun. \\ ${ }^{3}$ Université de Dschang, Faculté des sciences, Département de Biologie Animale, Laboratoire \\ de physiologie Animale et de phytopharmacologie B.P.67 Dschang-Cameroun. \\ *Corresponding author, B.P.136 Bafia-Cameroun, Tél. (237) 75765002 E-mail: gaple1976@yahoo.fr
}

\section{RESUME}

Maneb et Chlorpyrifos-Ethyl sont des pesticides utilisés en grande quantité dans les cultures vivrières au Cameroun. Pour évaluer les risques de toxicité et leur influence négative sur la biodiversité aquatique, des bioessais de toxicité aiguë sur des individus d'Oreochromis niloticus ont été réalisés séparément avec six concentrations $(0 ; 1,80 ; 1,85 ; 1,90 ; 1,95$ et $2 \mathrm{mg} / \mathrm{l})$ de Maneb et $(0 ; 0,15 ; 0,16 ; 0,17 ; 0,18$ et $0,19 \mathrm{mg} / \mathrm{l})$ de Chlorpyrifos-Ethyl en trois répétitions en milieux statiques dans des aquariums pendant $24 \mathrm{~h}$. Des mortalités minimales $(20,00 \pm 0,00 \%)$ et maximales $(100,00 \pm 0,00 \%)$ ont été enregistrées chez les alevins (taille moyenne 3,86 $\pm 0,04 \mathrm{~cm}$ et de poids moyen $0,74 \pm 0,02 \mathrm{~g}$ ) avec respectivement 1,85 et $2 \mathrm{mg} / \mathrm{l}$ de Maneb puis 0,15 et $0,19 \mathrm{mg} / \mathrm{l}$ de Chlorpyrifos-Ethyl. Des corrélations ( $\mathrm{r}=+0,96$ pour le Chlorpyrifos-Ethyl et $\mathrm{r}=+0,98$ pour le Maneb) ont été positives et fortes entre les taux de mortalité et les concentrations. La concentration létale 50\% pendant 24 heures d'exposition $\left(\mathrm{CL}_{50-24 \mathrm{~h}}\right)$ a été relativement élevée avec le Maneb $(1,93 \mathrm{mg} / \mathrm{l})$ et faible avec le Chlorpyrifos-Ethyl $(0,154 \mathrm{mg} / \mathrm{l})$ qui s'avère le pesticide le plus toxique et qui provoque de plus un habitus particulier chez les poissons survivants.

(c) 2009 International Formulae Group. All rights reserved.

Mots clés : Maneb, Chlorpyrifos-Ethyl, toxicité, $\mathrm{CL}_{50-24 \mathrm{~h}}$, Oreochromis niloticus.

\section{INTRODUCTION}

La population mondiale est passée de 3 milliards en 1960 à presque 6,6 milliards en 2007 (Domenach, 2007). L'une des conséquences de cette croissance démographique est le déficit alimentaire observé dans certaines régions du monde et en particulier en Afrique. Entre 1964 et 1980, le degré d'autonomie alimentaire a régressé par exemple de 3\% au Mali, de 5\% au Sénégal et de $27 \%$ en Somalie (Rudolf, 1986). Dans presque tous les pays africains, l'importation des produits alimentaires a augmenté. En
1981, l'Afrique a importé 15 millions de tonnes de céréales (FAO, 1982) et 25 ans plus tard, elle en importa 2,5 fois plus (FAO, 2007).

Pour résoudre les problèmes de déficit alimentaire à travers la promotion de l'autonomie alimentaire, les activités agricoles se sont intensifiées avec l'utilisation accrue dans les cultures de grandes quantités de pesticides. La Société des Bananerais de M’Bome et les Sociétés des Plantations Nouvelles de Penja au Cameroun en 2001 ont utilisé plus de 423 tonnes et 256000 litres de 
pesticides variés (Mpoh, 2002). Certes, le recours aux pesticides a fait augmenter significativement la quantité de la production agricole, mais en même temps cet usage aurait une influence négative sur la biodiversité aquatique (Monkiédjé, 2000). Ainsi, il est alors à craindre que les eaux de ruissellement des bassins versants en provenance des zones d'exploitations agricoles soient une source de contamination des plans d'eaux, avec pour conséquence la destruction des ressources aquatiques, dont les poissons (Bouba, 2002). Le présent travail révèle les effets de toxicité de deux pesticides très utilisés en cultures vivrières au Cameroun, le Maneb et le Chlorpyrifos-Ethyl, sur un poisson (Oreochromis niloticus) fréquemment utilisé en pisciculture.

\section{MATERIEL ET METHODES Matériel animal}

Un total de 520 Tilapia du Nil (Oreochromis niloticus) de taille moyenne $3,86 \pm 0,04 \mathrm{~cm}$ et de poids moyen 0,74 $\pm 0,02$ g ont été pêchés dans un étang de Santchou (Ouest-Cameroun), puis acclimatés en aquarium $\left(70 \times 30 \times 30 \mathrm{~cm}^{3}\right)$ pendant quatre semaines. L'eau de source $(\mathrm{pH}=6,6$; température $=20{ }^{\circ} \mathrm{C}$; dureté $=62 \mathrm{mg} / \mathrm{l} \mathrm{de}$ $\mathrm{CaCO}_{3}$; conductibilité $=630 \mu \mathrm{s} / \mathrm{cm}$; alcalinité $=75 \mathrm{mg} / \mathrm{l}$ de $\mathrm{CaCO}_{3}$ ) a été utilisée pour l'élevage. L'oxygénation a été assurée par une pompe à air de type Rena et le milieu a été entièrement renouvelé matin et soir par siphonage. Les alevins ont été nourris une fois par jour avec $15 \mathrm{~g}$ d'aliment complet pour poissons.

\section{Pesticides testés}

Le Maneb (Plantineb 80), est un fongicide de la famille des dithiocarbamates importé et distribué au Cameroun par la Société JACO. Sa matière active, éthylène bis (dithiocarbamate) de manganèse $a$ pour formule brute $\mathrm{C}_{4} \mathrm{H}_{6} \mathrm{~N}_{2} \mathrm{~S}_{4} \mathrm{Mn}$. Sa solubilité dans l'eau est estimée à $6 \mathrm{mg} / \mathrm{l}$ et son point de fusion est approximativement $192{ }^{\circ} \mathrm{C}$ (Kidd et James, 1991). Il est utilisé pour combattre la rouille précoce et tardive sur la pomme de terre (Solanum tuberosum), la tomate (Solanum lycopersium) et beaucoup d'autres fruits et légumes. La dose d'emploi varie de 2 à $2,5 \mathrm{~kg} /$ hectare. Chez certains animaux et l’Homme, le Maneb est généralement absorbé par voies buccale et cutanée. Il s'accumule principalement dans la thyroïde, les reins et le cœur où il perturbe non seulement leurs diverses fonctions, mais cause aussi des paralysies respiratoires pouvant conduire à la mort (Edouards et al., 1991).

Le Chlorpyrifos-Ethyl (Dursban 4), est un insecticide de la famille des organophosphates importé et distribué également au Cameroun par la Société JACO. Sa matière active, 0,0-phosphorothioate diéthylique de 03,5,6-trichloro-2-pyridyl a pour formule brute $\mathrm{C}_{9} \mathrm{H}_{11} \mathrm{Cl}_{3} \mathrm{NO}_{3} \mathrm{PS}$. Sa solubilité dans l'eau est estimée à $2 \mathrm{mg} / \mathrm{l}$ et son point de fusion est compris entre 41 et $44{ }^{\circ} \mathrm{C}$ (Kidd et James, 1991). Il est utilisé pour lutter contre les moustiques, les cancrelats, les puces et les poux. Il est aussi efficace comme insecticide sur le cotonnier, les fruits et les légumes. Il agit sur les parasites comme un poison de contact avec une certaine action nocive sur leur estomac. La dose d'emploi est de $25 \mathrm{ml}$ pour 15 litres d'eau. Chez certains animaux et chez l'Homme, le Chlorpyrifos-Ethyl est généralement absorbé par voies respiratoire, orale et/ou cutanée. Il affecte les systèmes respiratoire, cardiovasculaire et principalement nerveux par inhibition de l'acétylcholinestérase avec pour conséquences des convulsions, des paralysies et même la mort (Fabre et Truhaut, 1954).

\section{Conduite des bioessais de toxicité aiguë et Analyse Statistique}

L'aptitude physiologique des poissons a été vérifiée avec le dichromate de potassium $\left(\mathrm{K}_{2} \mathrm{Cr}_{2} \mathrm{O}_{7}\right)$ conformément aux normes de l'Association Française de Normalisation (AFNOR). En effet, 1 gramme de $\mathrm{K}_{2} \mathrm{Cr}_{2} \mathrm{O}_{7}$ a été solubilisé dans 1 litre d'eau distillée et 6 volumes $(0 ; 285 ; 290 ; 295 ; 300$ et $305 \mathrm{ml}$ de solution de $\mathrm{K}_{2} \mathrm{Cr}_{2} \mathrm{O}_{7}$ ) ont été prélevés puis introduits dans des aquariums numérotés de 1 à 6 contenant chacun dix alevins et différentes quantités (1litre ; $715 ; 710 ; 705 ; 700$ et 695 $\mathrm{ml})$ d'eau de source $(\mathrm{pH}=6,6$; température = $20{ }^{\circ} \mathrm{C}$; dureté $=62 \mathrm{mg} / \mathrm{l}$ de $\mathrm{CaCO}_{3}$; conductibilité $=630 \mu \mathrm{s} / \mathrm{cm}$; alcalinité $=75$ $\mathrm{mg} / \mathrm{l}$ de $\mathrm{CaCO}_{3}$ ) de manière à obtenir 6 concentrations escomptées $(0$; 285 ; 290 ; 295 ; 300 et $305 \mathrm{mg} / \mathrm{l}$ de $\left.\mathrm{K}_{2} \mathrm{Cr}_{2} \mathrm{O}_{7}\right)$.Une $\mathrm{CL}_{50}$ $24 \mathrm{~h}$ (concentration létale $50 \%$ pendant 24 heures d'exposition) de $298 \mathrm{mg} / \mathrm{l}$ a été obtenue. Cette valeur $\left(\mathrm{CL}_{50-24 \mathrm{~h}}=298 \mathrm{mg} / \mathrm{l}\right)$ est 
conforme aux normes AFNOR rapportées par Mpoh (2002), qui attestent que les poissons sont physiologiquement aptes à être utilisés pour les tests de toxicité aiguë si et seulement si la $\mathrm{CL}_{50-24 \mathrm{~h}}$ de $\mathrm{K}_{2} \mathrm{Cr}_{2} \mathrm{O}_{7}$ est comprise entre 175 et $355 \mathrm{mg} / \mathrm{l}$.

Les essais de toxicité aiguë avec le Maneb et le Chlorpyrifos-Ethyl ont été conduits séparément en milieux statiques avec trois répétitions selon la méthode utilisée par Mpoh (2002). A cet effet, dix poissons et 1 litre d'eau de source $(\mathrm{pH}=6,6$; température $=$ $20{ }^{\circ} \mathrm{C}$; dureté $=62 \mathrm{mg} / \mathrm{l}$ de $\mathrm{CaCO}_{3}$; conductibilité $=630 \mu \mathrm{s} / \mathrm{cm}$; alcalinité $=75$ $\mathrm{mg} / \mathrm{l}$ de $\mathrm{CaCO}_{3}$ ) ont été mis dans l'aquarium $\mathrm{N}^{\circ} 1$ (témoin ; $0 \mathrm{mg} / \mathrm{l}$ ) puis 1 litre du mélange solution mère de pesticide et eau de source dans les aquariums $\mathrm{N}^{\circ} 2$ à 6 , de manière à couvrir une gamme de 5 concentrations sollicitées $(1,80 ; 1,85 ; 1,90 ; 1,95$; et 2 mg/l de Maneb puis 0,$15 ; 0,16 ; 0,17 ; 0,18$ et 0,19 $\mathrm{mg} / \mathrm{l}$ de Chlorpyrifos-Ethyl). Dix poissons ont également été introduits dans chacun des aquariums $\mathrm{N}^{\circ} 2$ à 6 (contenant 998,2 ; 998,15 ; 998,10 ; 998,05 ; 998 ml d'eau pour le Maneb et 999,85 ; 999,84 ; 999,83 ; 999,82 ; 999,81 ml d'eau pour le Chlorpyrifos-Ethyl) et les morts ont été dénombrés après $24 \mathrm{~h}$ d'exposition.

- Mortalités : sont considérés comme morts les poissons immobiles et couchés sur le flanc après les tests de toxicité aiguë.

- CL ${ }_{50-24 h}$ : Calculée par la formule $\mathrm{CL}_{50-24 \mathrm{~h}}=$ $10^{\left.\left(\mathrm{X}^{+(5-} \mathrm{Y}^{\mathrm{w}}\right)^{/ / \mathrm{b}}\right)}$ Ramade (1992), où CL $\mathrm{CL}_{50-24 \mathrm{~h}}$ $=$ concentration létale $50 \%$ après 24 heures d'exposition, $\mathrm{X}$ et $\mathrm{Y}^{\mathrm{w}}=$ probits auxiliaires des concentrations et des taux de mortalité, $\mathrm{b}=$ coefficient de régression.
- Survivants aux nageoires anormalement disposées: les nageoires pectorales et ventrales perpendiculairement positionnées aux surfaces latérales et ventrales des poissons après les tests de toxicité aiguë ont été considérées comme étant anormalement disposées.

Le t-test de Student Fisher a été fait avec le logiciel SPSS 12.0 pour la comparaison des moyennes au seuil de signification 5\%.

\section{RESULTATS \\ Mortalité des poissons en fonction des concentrations et des pesticides}

Les taux de mortalité des poissons en fonction des concentrations et des pesticides testés après 24 h d'exposition sont présentés dans le Tableau 1. Il ressort qu'en dehors des lots témoins et de ceux ayant reçu 1,80 mg/l de Maneb, les poissons morts (alevins de $O$. niloticus) ont été enregistrés avec toutes les autres concentrations. En général, les taux de mortalité ont augmenté de manière significative $(p<0,05)$ en fonction des concentrations croissantes $(r=+0,96$ pour le Chlorpyrifos-Ethyl et $\mathrm{r}=+0,98$ pour le Maneb). Les concentrations maximales (2 $\mathrm{mg} / \mathrm{l}$ de Maneb et 0,19 mg/l de ChlorpyrifosEthyl) ont provoqué la mort de la totalité des poissons. Pendant que la plus faible concentration en Maneb (1,80 mg/l) n'a provoqué aucun décès, celle du ChlorpyrifosEthyl (0,15 mg/l) en a provoqué 20\%.

Influence du type de pesticide sur la $\mathrm{CL}_{50-24 \mathrm{~h}}$ La figure 3 présente la $\mathrm{CL}_{50}$ - 24h des deux pesticides utilisés. Elle a varié en

Tableau 1: Influence des concentrations du Maneb et du Chlorpyrifos-Ethyl sur la mortalité des poissons.

\begin{tabular}{|c|c|c|c|c|}
\hline \multirow{2}{*}{$\begin{array}{l}\text { Nombre } \\
\text { d'alevins } \\
\text { par lot }\end{array}$} & \multicolumn{2}{|c|}{ Maneb } & \multicolumn{2}{|c|}{ Chlorpyrifos-Ethyl } \\
\hline & $\begin{array}{c}\text { Concentrations } \\
\text { (mg/l) }\end{array}$ & $\begin{array}{c}\text { Mortalités } \\
\text { (\%) }\end{array}$ & $\begin{array}{c}\text { Concentrations } \\
(\mathrm{mg} / \mathrm{l})\end{array}$ & $\begin{array}{c}\text { Mortalités } \\
\text { (\%) }\end{array}$ \\
\hline 10 & 0 (témoin) & $0,00 \pm 0,00^{\mathrm{a}}$ & 0 (témoin) & $0,00 \pm 0,00^{\mathrm{a}}$ \\
\hline 10 & 1,80 & $0,00 \pm 0,00^{\mathrm{a}}$ & 0,15 & $20,00 \pm 0,00^{b}$ \\
\hline 10 & 1,85 & $20,00 \pm 0,00^{\mathrm{b}}$ & 0,16 & $60,00 \pm 0,00^{\mathrm{C}}$ \\
\hline 10 & 1,90 & $47,00 \pm 8,00^{c}$ & 0,17 & $73,20 \pm 8,00^{\mathrm{d}}$ \\
\hline 10 & 1,95 & $60,00 \pm 0,00^{\mathrm{d}}$ & 0,18 & $86,00 \pm 8,00^{\mathrm{e}}$ \\
\hline 10 & 2,00 & $100,00 \pm 0,00^{\mathrm{e}}$ & 0,19 & $100,00 \pm 0,00^{f}$ \\
\hline
\end{tabular}




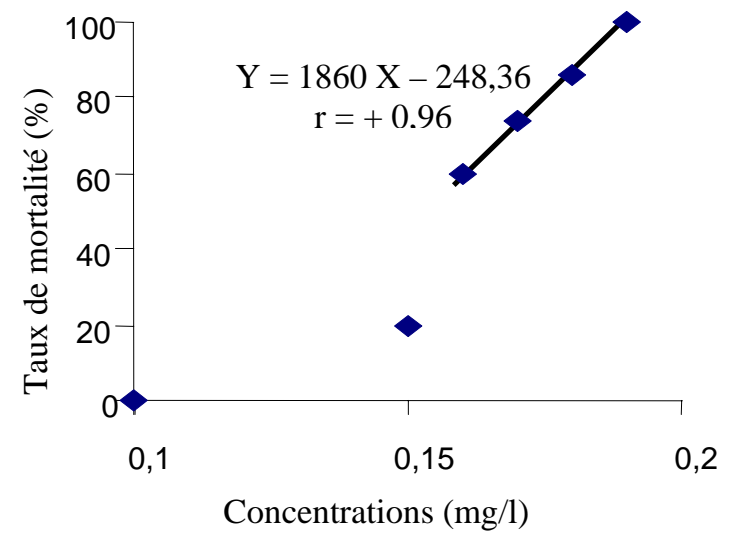

Figure 1 : Corrélation linéaire entre les taux de mortalité (Y) des alevins et les concentrations (X) du Chlorpyrifos-Ethyl.

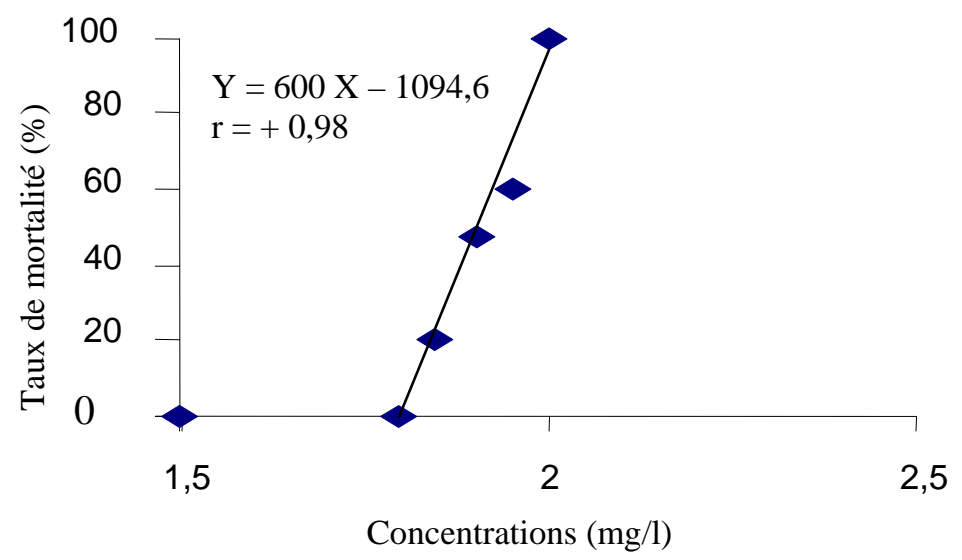

Figure 2 : Corrélation linéaire entre les taux de mortalité (Y) des alevins et les concentrations (X) du Maneb. mg/l : milligramme par litre ; \% : pourcentage

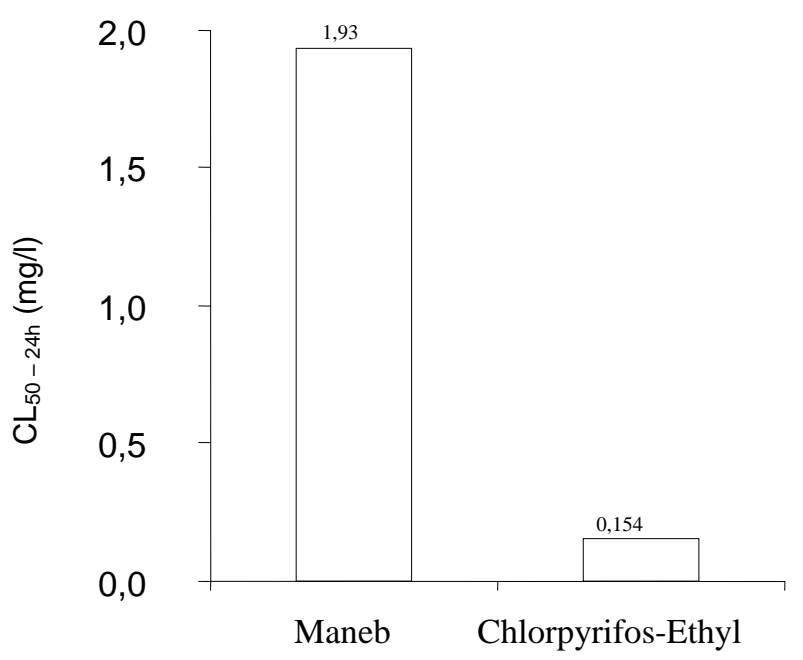

Figure 3 : $\mathrm{CL}_{50-24 \mathrm{~h}}$ des alevins en fonction du type de pesticide. 
fonction du type de pesticide. Elle a été de $1,93 \mathrm{mg} / \mathrm{l}$ pour le Maneb et $0,154 \mathrm{mg} / \mathrm{l}$ pour le Chlorpyrifos-Ethyl, soit environ 12,53 fois plus faible.

\section{Influence des pesticides sur l'habitus des poissons survivants}

Les effets du Maneb et du Chlorpyrifos-Ethyl en fonction des concentrations croissantes sur l'habitus des alevins survivants sont consignés dans le Tableau 2. Le Maneb n'a aucun effet sur la disposition habituelle des nageoires des poissons ayant survécus. Par contre, les survivants exposés au Chlorpyrifos-Ethyl, aux concentrations comprises entre 0,15 et 0,18 $\mathrm{mg} / \mathrm{l}$ ont eu toutes les nageoires pectorales et ventrales anormalement disposées.

\section{DISCUSSION}

L’étude a révélé à travers une augmentation croissante des taux de mortalité que le Maneb et le Chlorpyrifos-Ethyl sont de plus en plus toxiques aux alevins de $O$. niloticus aux concentrations croissantes. Ces résultats confirment ceux obtenus par Monkiédjé (2000) et Mpoh (2002) selon lesquels plusieurs pesticides sont toxiques pour les poissons et les invertébrés aquatiques à divers degrés. Ainsi, le Chlorpyrifos-Ethyl même à une concentration plus faible est plus toxique que le Maneb. Ceci serait dû aux différences de nature, de propriétés physicochimiques et de mode d'action des deux pesticides (Mpoh, 2002). En effet, les fongicides de la famille des dithiocarbamates (Maneb), malgré leurs actions perturbatrices du système endocrinien, sont peu solubles dans les lipides et très solubles dans l'eau (Fabre et Truhaut, 1954). Ils ont donc un faible potentiel d'accumulation dans les tissus organiques et seraient par conséquent facilement dilués et dispersés dans les systèmes aquatiques (Fabre et Truhaut, 1954). Par contre, les insecticides de la famille des organophosphates (Chlorpyrifos-Ethyl) ont une liposolubilité élevée et pénètrent plus aisément dans les tissus cutanés (Rice et al., 1997). Ils affectent ensuite le système nerveux par inhibition de l'acétylcholinestérase (Mpoh, 2002). L'accumulation excessive d'acétylcholine dans les voies nerveuses qui s'en serait suivie au niveau des synapses (Wikipédia, 2008) aurait inhibé par rétroaction, la libération de ce même neurotransmetteur dans le système nerveux central, empêchant ainsi la transmission de l'influx nerveux et causant la mort des organismes intoxiqués (Wikipédia, 2008).

En outre, la toxicité du ChlorpyrifosEthyl s'est révélée d'autant plus grande car quels que soient les niveaux de concentration testés, il engendre la déformation des nageoires pectorales et ventrales des poissons ayant survécus, contrairement au Maneb qui n’engendre aucune déformation. Les déformations observées avec le ChlorpyrifosEthyl seraient dues à une tétanisation qui est un état de contractions permanentes survenues dans les muscles des nageoires (Ahlem et al., 2007). En effet, chez ces survivants, la transmission de l'influx nerveux n'aurait pas été totalement supprimée. Mais l'inhibition d'un taux considérable d'acétylcholinestérase (>70\%) par le Chlorpyrifos-Ethyl a généré au

Tableau 2 : Influence des concentrations du Maneb et du Chlorpyrifos-Ethyl sur l'habitus des poissons survivants.

\begin{tabular}{|c|c|c|c|c|c|}
\hline \multicolumn{3}{|c|}{ Maneb } & \multicolumn{3}{|c|}{ Chlorpyrifos-Ethyl } \\
\hline $\begin{array}{l}\text { Conc. } \\
\text { (mg/l) }\end{array}$ & EAS & $\begin{array}{c}\text { SNAD } \\
(\%)\end{array}$ & $\begin{array}{l}\text { Conc. } \\
\text { (mg/l) }\end{array}$ & EAS & $\begin{array}{c}\text { SNAD } \\
(\%)\end{array}$ \\
\hline 0 (témoin) & $10,00 \pm 0,00$ & $0,00 \pm 0,00$ & 0 (témoin) & $10,00 \pm 0,00$ & $0,00 \pm 0,00^{\mathrm{a}}$ \\
\hline 1,80 & $10,00 \pm 0,00$ & $0,00 \pm 0,00$ & 0,15 & $8,00 \pm 00$ & $100,00 \pm 0,00^{b}$ \\
\hline 1,85 & $8,00 \pm 0,00$ & $0,00 \pm 0,00$ & 0,16 & $4,00 \pm 0,00$ & $100,00 \pm 0,00^{b}$ \\
\hline 1,90 & $5,34 \pm 0,34$ & $0,00 \pm 0,00$ & 0,17 & $2,68 \pm 0,47$ & $100,00 \pm 0,00^{b}$ \\
\hline 1,95 & $4,00 \pm 0,00$ & $0,00 \pm 0,00$ & 0,18 & $1,34 \pm 0,47$ & $100,00 \pm 0,00^{b}$ \\
\hline 2,00 & $0,00 \pm 0,00$ & / & 0,19 & $0,00 \pm 0,00$ & l \\
\hline
\end{tabular}


niveau des fentes des synapses neuromusculaires des nageoires l'accumulation d'une quantité d'acétylcholine pas excessive mais tout de même supérieure à la normale (Wikipédia, 2008). La surcharge d'acétylcholine aurait stimulé des violentes et permanentes contractions des muscles (Ahlem et al., 2007) qui, grâce à leurs tendons seraient probablement à l'origine des tractions sans cesse exercées sur les ceintures scapulaires et pelviennes des poissons et des modifications de la position initiale de leurs nageoires.

Le Maneb avec une $\mathrm{CL}_{50-24 \mathrm{~h}}=1,93$ $\mathrm{mg} / \mathrm{l}$ et le Chlorpyrifos-Ethyl avec une $\mathrm{CL}_{50}$ ${ }_{24 \mathrm{~h}}=0,154 \mathrm{mg} / \mathrm{l}$, sont toxiques pour les alevins de O. niloticus $\left(\mathrm{CL}_{50}<100 \mathrm{mg} / \mathrm{l}\right.$; Jacques et al., 1997). Le Chlorpyrifos-Ethyl ayant une $\mathrm{CL}_{50-24 h}$ faible s'avère plus toxique et déforme en plus les nageoires pectorales et ventrales des alevins survivants.

\section{REFERENCES}

Ahlem BS, Fethia HS, Wahida B, Nabil C, Mahjoub A. 2007. Effet de l'extrait méthanolique de Chrysanthemum trifurcatum (Desf.) Batt. et Trab. Sur la motricité duodénale de rat. Comptes Rendus Biologiques, 330 : 226-230.

Bouba O. 2002. Bio évaluation de la toxicité potentielle des lixiviats de sols en monoculture intensive du bananier dans le groupe des sociétés SPNP-SBM-PHP à Njombé, Cameroun. Thèse de Master, Université de Dschang, 54p.

Domenach H. 2007. Démographie et environnement: vers une régulation planétaire ? La Jaune et la Rouge, 1: 1117.

Edouards IR, Bac DG, Temple WA, Hayes WJ, Lois E. 1991. Fongicides et Composés Reliés, Manuel de la Toxicologie de Pesticide. Edition Académique: New York; 2-4.
Fabre R, Truhaut R. 1954. Toxicologie des Produits Phytopharmaceutiques. Société d'Edition d'Enseignement Supérieur SEDES: Paris ; 272p.

FAO (Organisation des Nations Unies pour l'Alimentation et l'Agriculture). 1982. La Situation mondiale de l'Alimentation et de l'Agriculture. FAO : Rome ; 164p.

FAO (Organisation des Nations Unies pour l'Alimentation et l'Agriculture). 2007. Perspectives de Récoltes et Situations Alimentaires. FAO : Rome ; 68 p.

Jacques D, Jean B, Jean CH. 1997. Physicochimie des Lubrifiants. Analyse et Essais. Technip: France ; 432p.

Kidd H, James DR. 1991. Le Manuel d’Agrochimique ( $3^{\mathrm{e}}$ édn). Société Royale des Services d'Information de Chimie: Cambrige; $3-4$.

Monkiédjé AB, Ngassam $\mathrm{P}$, Njiné $\mathrm{T}$, Demanou J, Kemba N, Zebaze SH. 2000. The response of plankton communities in laboratory microcosms to Ridomil plus 72, a heavily used fungicide in Cameroon. A. J. Sci. Technol., 1: 13-20.

Mpoh EJ. 2002. Bioévaluation de la toxicité de quelques pesticides Decis 25 EC, Gramoxone, Super et Vydate L utilisés en culture industrielle de la banane. Thèse de Master, Université de Dschang, 63p.

Ramade F. 1992. Précis d'Ecotoxicologie. Masson: Paris; 492 p.

Rice PJ, Drewes CD, Klubertanz TM, Bradbury SP, Coats JR. 1997. Acute toxicity and behavioral effects of Chlorpyrifos, permethrin, phenol, strychnine, and 2,4-dinitrophenol to 30days-old Japanese Medaka (Oryzias latipes). Setac. Journals, 16: 696-704.

Rudolf HS. 1986. Pourquoi Sont-ils Si Pauvres? (Nouvelle édn). Presses des Remparts à Yverdon : Suisse ; 219 p.

Wikipédia. 2008. Insecticide. http://fr. wikipédia.org/w/index.php. 